\title{
EXAMPLES OF WEAK BOUNDARY COMPONENTS
}

\author{
TOHRU AKAZA and KÔTARO OIKAWA
}

1. Let $D$ be a plane domain and $\Gamma$ be a component of the boundary of $D$ consisting of a single point. According to Sario [5] we shall call $\Gamma$ a weak boundary component of $D$ if its image under any conformal mapping of $D$ consists of a single point. A weak boundary component has been introduced by Grötzsch [2], who called it "vollkommen punktförmig". If $\Gamma$ is not weak we shall say that it is unstable (Sario [5]). We know that the weakness depends merely on the configuration of $D$ in a neighborhood of $\Gamma$ (see [4], p. 274).

Let $E$ be a compact set on the non-negative real axis such that $0 \in E, E C$ $[0,1]$, and that the component of 0 contains no other point. Let $h(\xi)$ be a real (finite) valued function which is defined on $E$, upper semi-continuous, nonnegative, and such that $h(0)=0$. For any $\xi \in E$, let

$$
S_{\xi, h}=\{z ; \operatorname{Re} z=\xi,|\operatorname{Im} z| \leqq h(\xi)\} .
$$

Then $D_{E, h}=\{z ;|z| \leqq \infty\}-\cup_{\Sigma \in E} S_{5, h}$ is a domain and $\Gamma_{F, h}=\{0\}$ is its boundary component consisting of a single point.

It would be useful to give convenient condition on $E$ and $h(\xi)$ to determine when $\Gamma_{k, h}$ is weak or unstable.

2. We remark first that the following "comparison theorem" would enlarge the range of applicability of criteria given in the sequel: If $\Gamma_{E, h_{1}}$ is weak and

$$
\varlimsup_{\xi \in E, \xi \rightarrow 0} \frac{h_{2}(\xi)}{h_{1}(\xi)}<\infty
$$

then $\Gamma_{E, h_{2}}$ is also weak. The proof is immediate from the local property and the quasi-conformal invariance of weakness (see [4], p. 274).

3. The former author has shown that, if $E=\left\{a_{n}\right\}_{n=1}^{\infty}\left(a_{n}>a_{n+1}>0\right.$, $\left.\lim _{n \rightarrow \infty} a_{n}=0\right)$ and $h(\xi) \leqq c \xi(c>0)$, then $\Gamma_{E, h}$ is weak (see [1]). It is generalized as follows (cf. the comparison theorem) :

Received December 15, 1960. 
Theorem 1. If $D_{F, h}$ and $\Gamma_{F, h}$ are given by the restriction onto $E$ of $a$ function $h(\xi)$ defined on $0 \leqq \xi \leqq 1$ such that

(i) $\sqrt{\xi^{2}+h(\xi)^{2}}$ is a non-decreasing function of $\xi$ with the derivative (existing almost every where) bounded away from zero,

$$
\int_{[0,1]-E \sqrt{\xi^{2}+h(\xi)^{2}}}=\infty,
$$

then $\Gamma_{E, h}$ is a weak boundary component.

Proof. Take $b>0$ such that $\{z ; b \leqq|z| \leqq \infty\} \subset D_{E, h}$. Let $\{\gamma\}$ be the family of all the closed rectifiable curves in $D_{E, h} \cap\{z ;|z|<b\}$ separating $\Gamma_{E, h}$ from $|z|=b$. It has been shown by Jurchescu [3] that $\Gamma_{E, h}$ is weak if and only if the extremal length $\lambda\{r\}$ of the family $\{r\}$ vanishes (see also [4], Theorems 2, 3).

For a $\xi \in[0,1]-E$, let $\gamma_{\text {s }}$ be the union of $\gamma_{\xi}^{\prime}=\{z ; \operatorname{Re} z=\xi,|\operatorname{Im} z| \leqq h(\xi)\}$ and $\gamma_{5}^{\prime \prime}=\left\{z ;|z|^{2}=\xi^{2}+h(\xi)^{2}, \arctan (h(\xi) / \xi) \leqq|\arg z| \leqq \pi\right\}$. Evidently $\left\{\gamma_{\xi}\right\}=$ $\left\{\gamma_{\xi} ; \xi \in[0,1]-E\right\}$ is contained in $\{\gamma\}$ and, therefore, it is sufficient to show that $\lambda\left\{r_{\xi}\right\}=0$. On making use of usual notations, we have

$$
L_{\rho}\left\{\gamma_{\xi}\right\}^{2} \leqq\left(\int_{r_{\xi}} \rho d s\right)^{2} \leqq\left(\int_{r_{\xi}} d s\right)\left(\int_{r_{\xi}} \rho^{2} d s\right)^{2} \leqq 2 \pi \sqrt{\xi^{2}+h(\xi)^{2}}\left(\int_{r_{\xi}^{\prime}} \rho^{2} d y+\int_{r_{\xi}^{\prime \prime}} \rho^{2} r d \theta\right) .
$$

where $r=\sqrt{\xi^{2}+h(\xi)^{2}}$. Divide it by $r$ and integrate it with respect to $\xi$ over $[0,1]-E$. Since it is assumed that $d r / d \xi \geqq \alpha>0$, we have, on putting $\Delta^{\prime}=U_{\xi} r_{\xi}^{\prime}$ and $\Delta^{\prime \prime}=U_{\xi} \gamma_{\xi}^{\prime \prime}$, that

$$
L_{\rho}\left\{r_{\xi}\right\}^{2} \int_{[0,1]-E} \frac{d \xi}{\xi^{2}+h(\xi)^{2}} \leqq 2 \pi \iint_{\Delta^{\prime}} \rho^{2} d x d y+\frac{2 \pi}{\alpha} \iint_{\Delta^{\prime \prime}} \rho^{2} r d r d \theta \leqq \operatorname{const} \iint_{D} \rho^{2} d x d y .
$$

Therefore, $L_{\rho}\left\langle r_{\xi}\right\}^{2}=0$ for any square integrable $\rho$, i.e., $\lambda\left\{r_{\xi}\right\}=0$.

4. A result of the former author [1] saying that $\Gamma_{F, h}$ is unstable when $E=\{1 / n\}_{n=1}^{\infty}$ and $h(\xi)=\xi^{p}(0<p<1)$ will be contained in the following:

TheOREM 2. If $D_{k, h}$ and $\Gamma_{E, h}$ are given by the restriction onto $E$ of a function $h(\xi)$ defined on $0 \leqq \xi \leqq 1$ such that

(i) monotone non-decriasing

(ii) there exists a constant $K$ such that, for any $\xi \in E-\{1\}$, it is possible to find $a \xi^{\prime} \in E$ with $\xi<\xi^{\prime}$ and $h\left(\xi^{\prime}\right) \leqq K h(\xi)$.

$$
\int_{[0,1]-\tau)} \frac{d \xi}{h(\xi)}<\infty,
$$


then $\Gamma_{E, h}$ is an unstable boundary component.

That the condition (ii) cannot be omitted for the case $\int_{0}^{1} d \xi / h<\infty$ is seen from an easily constructed example. Whether or not we can omit it for the case $\int_{0}^{1} d \xi / h=\infty$ is not clear, however, we can do so for $h(\xi)=\xi^{p}(p \geqq 1)$ as follows :

Corollary. If $h(\xi)=\xi^{p}(p \geqq 1)$, then $\Gamma_{k, h}$ is an unstable boundary component of $D_{R, h}$ provided

$$
\int_{[0,1]-E} \frac{d \xi}{\xi^{p}}<\infty
$$

Proof of Theorem 2. We shall apply the following criterion due to Grötzsch ([2]; see also [4]. Theorem 3): Take $b$ such that $\{z ; b \leqq|z| \leqq \propto\} \subset D_{k, h}$; $\Gamma_{E, h}$ is unstable if and only if there exists a finite number $M$ such that $\sum_{\nu=1}^{k} \bmod A_{\nu} \leqq M$ holds for any finite set $\left\{A_{1}, A_{2}, \ldots, A_{k}\right\}$ of doubly connected domains $A_{\nu}$ with the following conditions:

$$
\begin{aligned}
& \text { (1) } A_{\nu} \subset D_{E, h} \cap\{z ;|z|<b\}, \\
& \text { (2) } A_{\nu} \text { separates } \Gamma_{E, h} \text { from }|z|=b \text {, } \\
& \text { (3) } A_{\nu} \text { separates } A_{\nu-1} \text { from } A_{\nu+1} .
\end{aligned}
$$

On looking over the argument in [4] we understand that the result is true if every $A_{\nu}$ is so restricted that the boundary consists of closed analytic curves.

Let $(0, b)-E=\cup_{n=1}^{\infty} I_{n}$, where $I_{n}=\left(\xi_{n}, \xi_{n}^{\prime}\right)$ are mutually disjoint open intervals. Consider the quadrilaterals $Q_{n}=\left\{z ; \operatorname{Re} z \in I_{n},|\operatorname{Im} z| \leqq h\left(\xi_{n}\right)\right\} \quad(n=$ $1,2, \ldots)$. By the condition (2) every $A_{\nu}$ passes through a $Q_{n}$ vertically, i.e., every closed arc in $A_{\nu}$ separating its boundary components contains a subarc connecting in $Q_{n} \cap A$, the top and the bottom sides of $Q_{n}$. There may be more than one $Q_{n}{ }^{\prime} s$; we then take the $Q_{n}$ corresponding to the left most $I_{n}$ (remember that the boundary of $A_{\nu}$ consists of analytic curves). For a $Q_{n}$, consider all the $A_{v}{ }^{\prime} s$ with the above property. Then, by the condition (3), the sum of their moduli does not exceed $2 \pi / \lambda\{\gamma\}_{n}$, where $\{\gamma\}_{n}$ is the family of all the closed curves in $D_{E, h} \cap\{z ;|z|<b\}$ which separate $\Gamma_{F, h}$ from $|z|=b$ and pass through $Q_{n}$ vertically.

We thus have a grouping of the set $\left\{A_{1}, A_{2}, \ldots, A_{k}\right\}$ in terms $Q_{n}$. Since an $A_{\nu}$ does not appear in different groups, $\sum_{\nu=1}^{k} \bmod A_{\nu} \leqq 2 \pi \sum_{n=1}^{\infty} 1 / \lambda\{\gamma\}_{n}$. 
Evidently $\lambda\{r\}_{n}$ is not less than mod $Q_{n}$, the "vertical" modulus of the quadrilateral $Q_{n}$, which is equal to $h\left(\xi_{n}\right) /\left(\xi_{n}^{\prime}-\xi_{n}\right)$. We conclude, on using the condition (ii) that

$$
\sum_{\nu=1}^{k} \bmod A_{\nu} \leqq 2 \pi \sum_{n=1}^{\infty} \frac{\xi_{n}^{\prime}-\xi_{n}}{h\left(\xi_{n}\right)} \leqq 2 \pi K \sum_{n=1}^{\infty} \frac{\xi_{n}^{\prime}-\xi_{n}}{h\left(\xi_{n}^{\prime}\right)} \leqq 2 \pi K \int_{[0, b]-F} \frac{d \xi}{h(\xi)}<\infty
$$

and that $I_{F, h}^{\prime}$ is unstable.

Proof of Corollary. Since

$$
\sum_{n=1}^{\infty}\left(1-\frac{\xi_{n}}{\xi_{n}^{\prime}}\right) \leqq \sum_{n=1}^{\infty} \int_{\xi_{n}}^{\xi_{n^{\prime}}} \frac{d \xi}{\xi^{p}}=\int_{[0, b]-E} \frac{d \xi}{\xi^{p}}<\infty,
$$

$\left(\xi_{n}^{\prime}\right)^{p} /\left(\xi_{n}\right)^{p}$ is bounded. This fact plays the role of (ii) in the above proof.

5. In a paper of the latter author, we proved the following ([4], Theorem 8):

Consider in particular $E=\{0\}^{\cup} \cup_{n=1}^{\infty}\left[u_{n}, u_{n}^{\prime}\right]$, where $0<u_{n}<u_{n}^{\prime}<u_{n-1}<1$ $(n=2,3, \ldots)$ and $\lim _{n \rightarrow \infty} u_{n}=0$. Then, under the assumption that $\lim _{n \rightarrow \infty}$ $\left(u_{n} / u_{n+1}^{\prime}\right)=1$ and $u_{n} / u_{n+1} \geqq 1+\delta>1$, the $\Gamma_{k, h}$ for $h \equiv 0$ is weak if and only if

$$
\sum_{n=1}^{\infty} \frac{1}{\log \frac{\boldsymbol{u}_{n+1}^{\prime}}{u_{n}-\boldsymbol{u}_{n+1}^{\prime}}}=\infty .
$$

Concerning such an $E$, Theorem 1 is merely saying that $\Gamma_{E, h}$ for $h \equiv 0$ is weak if $\sum_{n=1}^{\infty}\left(\left(u_{n} / u_{n+1}^{\prime}\right)-1\right)=\infty$. Theorem 2 is not applicable to the case where $h \equiv 0$. We see that there is a wide room into which our Theorems 1 and 2 should be extended.

6. Our theorems, however, may be extended into a different direction. To show this, we first introduce the notations

$$
S(\xi, \theta, c)=\{z ;|z+c|=\xi+c,|\arg z| \leqq \theta\} \quad(0 \leqq c<\infty, 0 \leqq \theta<\pi, 0<\xi)
$$

and

$$
S(\xi, \theta, \infty)=\{z ; \operatorname{Re} z=\xi,|\operatorname{Im} z| \leqq \xi \tan \theta\} \quad(0 \leqq \theta<\pi / 2,0<\xi) .
$$

Let $E$ be, as before, a compact set on the non-negative real axis such that $0 \in E, E \subset[0,1]$, and that the component of 0 contains no other point. Let $\theta(\xi)$ and $c(\xi)$ be functions which are defined on $E$ and satisfy the following conditions: $\theta(\xi)$ is upper semi-continuous and is such that $0 \leqq \theta(\xi)<\pi ; c(\xi)$ 
is continuous, non-decreasing, and is such that $0 \leqq c(\%) \leqq \infty$. Suppose further that $\theta(\xi)<\pi / 2$ whenever $c(\xi)=\infty$, and that

$$
\begin{array}{ll}
\lim _{\xi \in E, \xi \rightarrow 0} \theta(\xi) \leqq \frac{\pi}{2} & \text { if } 0<\lim _{\xi \in E, \xi \rightarrow 0} c(\xi)<\infty, \\
\lim _{\xi \in F, \xi \rightarrow 0} \xi \tan \theta(\xi)=0 & \text { if } c(\xi) \equiv \infty .
\end{array}
$$

Then

$$
D(E, \theta, c)=\{z ; 0<|z| \leqq \infty\}-\bigcup_{\xi \in E-(0)} S(\xi, \theta(\xi), c(\xi))
$$

is a domain and $\Gamma(E, \theta, c)=\{0\}$ is its boundary component. The domain $D_{E, h}$ discussed in the previous sections is the $D(E, \theta, c)$ for $\theta(\xi)=\arctan (h(\xi) / \xi)$ and $c(\xi) \equiv \infty$.

Theorem 1'. Suppose that $D(E, \theta, c)$ and $\Gamma(E, \theta, c)$ described above are given by restrictions onto $E$ of $\theta(\xi)$ and $c(\xi)$ defined on $0 \leqq \xi \leqq 1$, where $c(\xi)$ is non-decreasing on $0 \leqq \xi \leqq 1$. If either

(I) $\lim _{\xi \rightarrow 0} c(\xi)=0$ and

$$
\int_{[0,1]-E \xi+c(\xi)}^{d \xi}=\infty
$$

or

(II) the distance $r(\xi)$ between 0 and the endpoints of $S(\xi, \theta(\xi), c(\xi)$ ) is a non-decreasing function of $\xi$ with the derivative bounded away from zero and

$$
\int_{[0,1]-E)} \frac{d \xi}{r(\xi)}=\infty
$$

then $\Gamma(E, \theta, c)$ is weak.

Proof. If (I) is assumed, we may suppose without loss of generality that $c(\xi)$ is finite. The weakness of $\Gamma(E, \xi, c)$ follows from the vanishing of the extremal length of the family $\left\{_{\xi} ; \xi \in[0,1]-E\right\}$, where $\gamma_{\xi}=\{z ;|z+c(\xi)|=$ $\xi+c(\xi)\}$. Under the supposition of (II), we similarly consider $\left\{\gamma^{\prime} \cup \gamma_{\xi}^{\prime \prime} ; \xi \in[0,1]\right.$ $-E\}$, where $\gamma_{\xi}^{\prime}=S(\xi, \theta(\xi), c(\xi))$ and $\gamma_{\bar{\xi}}^{\prime \prime}=\{z ;|z|=r(\xi), \theta(\xi) \leqq|\arg z| \leqq \pi\}$. The proof in detail will be omitted since it is completely analogous to that of Theorem 1.

TheOREm 2'. Suppose that $D(E, \theta, c)$ and $\Gamma(E, \theta, c)$ described above are given by restrictions onto $E$ of $\theta(\xi)$ and $c(\xi)$ defined on $0 \leqq \xi \leq 1$, where $c(\xi)$ is non-decreasing on $0 \leqq \xi \leqq 1$. If 
(i) the length $l(\xi)$ of $S(\xi, \theta(\xi), c(\xi))$ is a non-decreasing function of $\xi$ provided that $c(\xi) \equiv \infty$, and $l(\xi) /(\xi+c(\xi))$ is non-decreasing otherwise,

(ii) there exists a constant $K$ such that, for any $\xi \in E-\{1\}$, it is possible to find $a \xi^{\prime} \in E$ with $\xi<\xi^{\prime}$ and $l\left(\xi^{\prime}\right) \leqq K l(\xi)$,

(iii)

$$
\int_{[0,1]-F} \frac{d(\xi+c(\xi))}{l(\xi)}<\infty
$$

where it is regarded that $d c(\xi) \equiv 0$ on the interval on which $c(\xi) \equiv \infty$, then $\Gamma(E, \theta, c)$ is unstable.

Proof is completely similar to that of Theorem 2. We shall just indicate the estimation of the modulus of the quadrilateral $Q$ defined by the domain bounded by $C_{\xi}=\{z ;|z+c(\xi)|=\xi+c(\xi)\}, \quad C_{\xi^{\prime}}=\left\{z ;\left|z+c\left(\xi^{\prime}\right)\right|=\xi^{\prime}+c\left(\xi^{\prime}\right)\right\}$, $\{z ; \arg z=l(\xi) /(\xi+c(\xi))\}$, and $\{z ; \arg z=-l(\xi) /(\xi+c(\xi))\}$ where $\xi<\xi^{\prime}$ and $c\left(\xi^{\prime}\right)<\infty$. Map the interior of $C_{\xi}$, onto $|\zeta|<1$ by a linear transformation which maps $C_{\xi}$ onto the circle $|\zeta|=a<1$. An elementary estimation of non-euclidean quantities shows that $a>\left(\xi+c\left(\xi^{\prime}\right)\right) /\left(\xi^{\prime}+c\left(\xi^{\prime}\right)\right)$ and that the image of $Q$ contains $\{\zeta ; 1 / a<|\zeta|<1,|\arg \zeta|<l(\xi) /(\xi+c(\xi))\}$. We conclude that

$\bmod Q \leqq \frac{\xi+c(\xi)}{l(\xi)} \log \frac{\xi^{\prime}+c\left(\xi^{\prime}\right)}{\xi+c(\xi)} \leqq \frac{\xi^{\prime}-\xi+c\left(\xi^{\prime}\right)-c(\xi)}{l(\xi)} \leqq K \int_{\xi}^{\xi^{\prime}} \frac{d(\xi+c(\xi))}{l(\xi)}$

\section{REFERENCES}

[1] Akaza, T., On the weakness of some boundary components. Nagoya Math. J., 17 (1960), 219-223.

[2] Grötzsch, H., Eine Bemerkung zum Koebeschen Kreisnormierungsprinzip. Ber. Verh. Sächs. Akad. Wiss. Leipzig. Math.-Nat. K1., 87 (1935), 319-324.

[ 3 ] Jurchescu, M., Modulus of a boundary component. Pacific J. Math., 8 (1958), 791-809.

[4] Oikawa, K., On the stability of boundary components. Ibid., 10 (1960), 263-294.

[5] Sario, L., Strong and weak boundary components. J. Anal. Math. 5 (1956/57), 389398.

Kanazawa University, Nagoya University

and Hirosima University 